\title{
The Quality of Numerical Software: Assessment and Enhancement
}

In this chapter we provide some background on the conference which brought together the researchers whose work is described in this volume. Further information, including access to transparencies and audio recordings of some of the talks, are available at the conference World Wide Web site: http://renoir.csc.ncsu.edu/IFIP/WoCo7/.

\section{CONFERENCE VENUE}

The conference entitled The Quality of Numerical Software: Assessment and Enhancement, was held 8-12 July 1996 at St. Catherine's College in Oxford, England. This was the seventh working conference organized by the IFIP Working Group on Numerical Software (WG 2.5) on behalf of the IFIP Technical Committee on Software: Theory and Practice (TC 2). The 76 conference attendees heard 26 invited presentations by researchers active in this field*. In addition, nine brief presentations were contributed by attendees in an "Open Session" which was arranged at the meeting itself. Considerable time was alotted for discussions of issues raised in the presentations, and the bulk of this discussion is recorded in this volume.

\section{THE PROGRAM}

\section{Monday, 8 July 1996}

9.00-9.30 Welcome, Administrative Details, Conference Rationale. Brian Ford (Numerical Algorithms Group, United Kingdom) and John Rice (Purdue University, USA)

9.30-10.10 Testing the Correctness of Linear Algebra Software. Nick Higham (University of Manchester, United Kingdom)

10.10-10.40 Break

10.40-11.20 Matrix Market: A Web Resource of Test Matrix Collections. Ronald F. Boisvert (National Institute of Standards and Technology, USA)

11.20-12.00 Is Nonnormality a Serious Computational Difficulty in Practice? Francoise Chatelin (University of Paris IX Dauphine, France)

12.00-12.20 Discussion of Morning Papers. Takao Tsuda (Hiroshima City University, Japan)

12.20-2.00 Lunch

\footnotetext{
*This proceedings volume contains two additional invited papers whose authors were unable to be present at the conference.
} 
2.00-2.40 Case Studies on the Development of ScaLAPACK and the NAG Numerical PVM Library. Sven Hammarling (Numerical Algorithms Group, United Kingdom)

2.40-3.20 Automatic Parallel Program Generation for Finite Element Analysis. Shun Doi (NEC Corp., Japan)

3.20-3.50 Break

3.50-4.30 Improving the Usability of Numerical Software through User-Centered Design. Cherri Pancake (Oregon State University, USA)

4.30-5.10 Network-based Scientific Computation via Inferno: Solution of Partial Differential Equations. Bill Coughran, Jr (Bell Laboratories, USA)

5.10-5.30 Discussion of Afternoon Papers. Bo Einarsson (University of Linkoping, Sweden)

\section{Tuesday, 9 July 1996}

9.00-9.40 Quality of Service and Scientific Workflows. Mladen Vouk (North Carolina State, USA)

9.40-10.20 Organizing a Numerical Software Development Team. Jeremy Du Croz (Numerical Algorithms Group, United Kingdom)

10.20-10.50 Break

10.50-11.30 Assuring the Quality of the Process of Assuring Software Quality. Lee Osterweil (University of Massachusetts, USA)

11.30-12.10 Real Inferno. Eric Grosse (Bell Laboratories, USA)

12.10-12.30 Discussion of Morning Papers. John Rice (Purdue University, USA)

12.30-2.10 Lunch

2.10-2.50 Testing and Evaluating Software for Large-Scale Constrained Optimization. Philip Gill (University of California at San Diego, USA)

2.50-3.30 Automatic Differentiation and Numerical Software Design. Chris Bischof (Argonne National Laboratory, USA)

3.30-3.50 Break

3.50-4.30 The Impact of Automatic Differentiation and Result Verification Techniques on the Quality of Numerical Software. Wolfgang Walter (Technical University Dresden, Germany)

4.30-5.10 The Visual Diagnosis on Numerical Calculation of PDE Problems and Experiments. Yukio Umetani (Hitachi, Japan)

5.10-5.30 Discussion of Afternoon Papers. Margaret Wright (Bell Laboratories, USA)

\section{Wednesday, 10 July 1996}

9.00-9.40 Developing ODE Software in New Computing Environments. Larry Shampine (Southern Methodist University, USA)

9.40-10.20 The Reliability of Standard Local Error Control Algorithms for Initial Value Ordinary Differential Equations. Des Higham (University of Dundee, United Kingdom)

10.20-10.50 Break 
10.50-11.30 The Evaluation of Numerical Software for Delay Differential Equations. Wayne Enright (University of Toronto, Canada)

11.30-12.10 Development of Efficient General Purpose Monte Carlo Codes Used in Nuclear Engineering. Masayuki Nakagawa (Japan Atomic Energy Research Institute)

12.10-12.30 Discussion of Morning Papers. Ian Gladwell (Southern Methodist University, USA)

\section{Thursday, 11 July 1996}

9.00-9.40 Testing Functions of One or Two Arguments. Van Snyder (Jet Propulsion Laboratory, USA)

9.40-10.20 The T-Experiments: Errors in Scientific Software. Les Hatton (Programming Research Limited, United Kingdom)

10.20-10.50 Break

10.50-11.15 Visualisation Benchmarking. Jeremy Walton (Numerical Algorithms Group, United Kingdom)

11.15-11.55 A Methodology for Testing the Numerical Correctness of Approximation and Optimisation Software and Examples of Its Application. Maurice Cox (National Physical Laboratory, United Kingdom)

11.55-12.15 Discussion of Morning Papers. Eric Grosse (Bell Laboratories, USA)

12.15-2.00 Lunch

\section{OPEN SESSION}

2.00-2.20 Performance Profile for Optimization. James Lyness (Argonne National Laboratory, USA)

2.20-2.40 Godess - A Platform to Compare ODE-methods. Per Grove Thomsen (Danish Technical University)

2.40-3.00 Godess - A Generic ODE Solving System. Gustaf Soederlind (Lund University, Sweden)

3.00-3.20 Computing Space-Time Curvature Using Higher Order Spatial Approximations. William Schiesser (Lehigh University, USA)

3.20-3.40 Break

3.40-4.00 Software Test System for Special Functions. Dan Lozier (National Institute of Standards and Technology, USA)

4.00-4.20 Parallel Globally Adaptive Integration. Ian Gladwell (Southern Methodist University, USA)

4.20-4.40 Superconductor Simulation. Mo Mu (Hong Kong University of Science and Technology)

4.40-5.00 A Programming Environment for Scientific Computing. Siegfried Rump (Technical University of Harburg, Germany)

5.00-5.20 Why We Couldn't Use Numerical Libraries for PETSc. Bill Gropp (Argonne National Laboratory, USA)

5.20-5.40 Discussion of Afternoon Presentations. Mladen Vouk (North Carolina State University, USA) 


\section{Friday, 12 July 1996}

9.00-9.40 Two Approaches to Exception Handling in Fortran. John Reid (Rutherford Appleton Laboratory, United Kingdom)

9.40-10.20 The XSC-Tools for Extended Scientific Computing. Ulrich Kulisch (University of Karlsruhe, Germany)

10.20-10.50 Break

10.50-11.30 Is Numerical Software Relevant? -Is it too late to worry about quality? James C.T. Pool (California Institute of Technology, USA)

11.30-12.10 If Software Quality is a Perception, How do we Measure It? Morven Gentleman (National Research Council, Canada)

12.10-12.30 Discussion of Morning Papers. Brian Smith (University of New Mexico, USA)

\section{ORGANIZATION}

\section{Program Committee}

Brian Ford (Oxford, United Kingdom) Co-chair

John Rice (West Lafayette, Indiana, USA), Co-chair

Franoise Chaitin-Chatelin (Toulouse, France)

Jeremy DuCroz (Oxford, United Kingdom)

Bo Einarsson (Linkping, Sweden)

Wayne Enright (Toronto, Ontario, Canada)

Eric Grosse (Murray Hill, New Jersey, USA)

Richard Hanson (Houston, Texas, USA)

Elias Houstis (Patras, Greece)

George Paul (Yorktown Heights, New York, USA)

Takou Tsuda (Hiroshima, Japan)

Mladen Vouk (Raleigh, North Carolina, USA)

\section{Local Arrangements}

Brian Ford

Frances Iles

Jeremy Du Croz

Stephen Hague

Sarah Naisby

Ian Reid 


\section{ATTENDEES}

Christian Bischof (Argonne National Laboratory, USA)

Petter Bjorstad (University of Bergen, Norway)

Ronald Boisvert (National Institute of Standards and Technology, USA)

Richard Brankin (Numerical Algorithms Group Ltd., United Kingdom)

Francoise Chaitin-Chatelin (CERFACS, France)

George Corliss (Marquette University, USA)

William M. Coughran (Lucent Technologies, Bell Laboratory Innovations, USA)

Maurice Cox (National Physical Laboratory, United Kingdom)

Pasqua D'Ambra (Center for Research on Parallel Computing and Supercomputing, Italy)

Luisa D'Amore (Center for Research on Parallel Computing and Supercomputing, Italy)

Daniela Di Serafino (Second University of Naples and Center for Research on Parallel

Computing and Supercomputing, Italy)

Jacques De Swart (Centre for Mathematics and Computer Science, France)

Shun Doi (NEC Corporation, Japan)

Jeremy Du Croz (Numerical Algorithms Group Ltd., United Kingdom)

Bo Einarsson (University of Linkoping, Sweden)

Wayne Enright (University of Toronto, Canada)

Graeme Fairweather (Colorado School of Mines, USA)

Brian Ford (Numerical Algorithms Group Ltd., United Kingdom)

Lloyd Fosdick (University of Colorado, USA)

T. Len Freeman (University of Manchester, United Kingdom)

Andreas Frommer (University of Wuppental, Germany)

Patrick Gaffney (Bergen Software Services International A/S, Norway)

W. Morven Gentleman (National Research Council, Canada)

Philip Gill (University of California at San Diego, USA)

Ian Gladwell (Southern Methodist University, USA)

William Gropp (Argonne National Laboratory, USA)

Eric Grosse (Bell Laboratories, USA)

Steve Hague (Numerical Algorithms Group Ltd., United Kingdom)

Sven Hammarling (University of Tennessee, USA)

Richard Hanson (Visual Numerics, Germany)

Les Hatton (Programming Research Ltd., United Kingdom)

Pieter W. Hemker (CWI, Netherlands)

Desmond J. Higham (University of Dundee, United Kingdom)

Nicholas Higham (University of Manchester, United Kingdom)

Tim Hopkins (University of Kent, United Kingdom)

Peter Jensen (Siemens A/S Telecom, Denmark)

Ulrich Kulisch (University of Karlsruhe, Germany)

Reino Kurki-Suonio (Tampere University of Technology, Finland)

Ulrich Langer (Johannes Kepler University, Germany)

Raytcho Lazarov (Texas A and M University, USA)

Daniel Lozier (National Institute of Standards and Technology, USA)

James Lyness (Argonne National Laboratory, USA)

Alan McCoy (CERFACS, France) 
Mo Mu (Hong Kong University of Science and Technology, Hong Kong)

Paul Muir (Saint Mary's University, Canada)

Almerico Murli (Center for Research on Parallel Computing and Supercomputing, Italy)

Masayki Nakagawa (Japan Atomic Energy Research Institute, Japan)

Leon Osterweil (University of Massachusetts, USA)

Cherri Pancake (Oregon State University, USA)

George Paul (IBM T.J. Watson Research Center, USA)

Ivor Philips (Boeing, USA)

James Pool (California Institute of Technology, USA)

John Pryce (Royal Military College of Science, United Kingdom)

John Reid (Rutherford Appleton Laboratory, United Kingdom)

John Rice (Purdue University, USA)

Siegfried M. Rump (Technical University of Hamburg-Harburg, Germany)

Venkat Sastry (Royal Military College of Science, United Kingdom)

Michael Saunders (Stanford University, USA)

William E. Schiesser (Lehigh University, USA)

Lawrence Shampine (Southern Methodist University, USA)

Masaaki Shimasaki (Kyushu University, Japan)

Brian Smith (University of New Mexico, USA)

Gustaf Soderlind (Lund University, Sweden)

Hans J. Stetter (Technical University of Vienna, Austria)

Per Thomsen (Denmark Technical University, Denmark)

Michael Thune (Uppsala University, Sweden)

Takao Tsuda (Hiroshima City University, Japan)

Christoph Ueberhuber (Technical University of Vienna, Austria)

Yukio Umetani (Hitachi Ltd., Japan)

W. Van Snyder (Jet Propulsion Laboratory, USA)

Heinrich Voss (Technical University of Hamburg-Harburg, Germany)

Mladen Vouk (North Carolina State University, USA)

Wolfgang Walter (Technical University of Dresden, Germany)

Jeremy Walton (Numerical Algorithms Group Ltd., United Kingdom)

Margaret Wright (Bell Laboratories, USA)

Stephen Wright (Argonne National Laboratory, USA) 